\title{
Aortic Surgery Symposium 2012 discussions-Panel 1 (sessions I and II): Aortic root, ascending aorta, and transcatheter aortic valve implantation (TAVI)
}

\author{
Moderator: Nicholas T. Kouchoukos, MD, St. Louis, Mo. \\ Panelist: Dianna M. Milewicz, MD, University of Texas Medical School at Houston, Houston, Texas; Alan C. \\ Braverman, MD, Washington University School of Medicine, St. Louis, Mo; Michael Mack, MD, Baylor Health \\ Care System, Plano, Texas; Lars G. Svensson, MD, Cleveland Clinic, Cleveland, Ohio; Rüdiger Lange, MD, \\ Germany Heart Center, Munich, Germany; Tirone E. David, MD, Toronto General Hospital, Toronto, Ontario, \\ Canada; Gebrine El Khoury, MD, Université Catholique de Louvain (UCL), Brussels, Belgium; Hans-Joachim \\ Schäfers, MD, University Hospital of Saarland, Homburg/Saar, Germany; Duke E. Cameron, MD, Johns Hop- \\ kins Hospital, Baltimore, Md; Fabian A. Kari, MD, Cardiothoracic Surgery, Stanford University Medical \\ School, Stanford, Calif; Christian D. Etz, MD, Cardiovascular Surgery, Heart Center Leipzig, Leipzig, Germany.
}

\section{Discussion}

Dr Kouchoukos. We will now proceed to the panel discussion, which will include the presenters from the first and second sessions. We have covered a number of diverse topics in both sessions and will encourage questions from the audience. Please speak up and challenge the people on the panel with some good questions. I will start out with a few just to get the discussion going.

Dr Milewicz, in patients who have the familial and sporadic type of dissections, whom do you screen with genetic testing and with imaging?

Dr Milewicz. If you have patients with familial disease, it is inherited in an autosomal dominant manner. There is a 50/50 risk that they will pass on the gene to their children. So the children need to be screened, and then the siblings need to be screened, and then whichever parent carried the mutation, their relatives need to be tracked down and screened.

So you can see that once you know the gene mutation, you can exclude $50 \%$ of the relatives for follow-up. Once you have the gene, it becomes very powerful for determining who is at risk. The individuals with a family history probably need to be routinely screened every 3 to 5 years, even if they have a normal aorta, until the gene for the family is identified.

Dr Kouchoukos. Do you use genetic testing for all these patients? This is expensive! Can you tell us how you implement that?

Dr Milewicz. The costs are falling rapidly. Right now, if you order each gene individually, it is quite expensive. They just started to set up panels of all the genes that we have identified, and that is less than the cost of computed tomography (CT) or magnetic resonance imaging. So, in the big picture of things, it is a relatively minimal cost, because once you have the gene, you can go through

Disclosures: Authors have nothing to disclose with regard to commercial support. Read at The American Association for Thoracic Surgery Aortic Symposium, New York, New York, April 26-27, 2012.

Address for reprints: Eva B. Griepp, MD, Department of Cardiothoracic Surgery, Mount Sinai School of Medicine, New York, NY 10029 (E-mail: ebgriepp@aol. com).

J Thorac Cardiovasc Surg 2013;145:S48-55

$0022-5223 / \$ 36.00$

Copyright (c) 2013 by The American Association for Thoracic Surgery http://dx.doi.org/10.1016/j.jtcvs.2012.11.080 and screen other family members. Once you know the gene mutation, that screening costs about $\$ 200$ to $\$ 300$. So you can identify who is at risk, and cut down the amount of imaging you are doing in the family. Knowing the gene also helps inform the management of the affected individuals.

Dr Kouchoukos. Along those same lines for Dr Braverman, with regard to follow-up imaging in patients who have had surgical procedures or even those who you are following who have not had surgery, what is your preferred method of screening? We are getting pressure, as most of you know, from some of the monitoring agencies about the cost, and also the danger in terms of radiation with computed tomographic imaging. So how are we going to deal with that problem?

Dr Braverman. I usually recommend magnetic resonance screening in follow-up of individuals to avoid the radiation exposure over the long term. To echo what Dianna said with regard to the cost of doing mutation analysis in patients who have Marfan syndrome or Loeys-Dietz syndrome clinically, and then the other family members: once an insurance company denies coverage for doing gene testing, it often requires a phone call to the medical director or someone higher up in the organization to explain the cost of continued surveillance over time. They will often change their minds and then allow mutation analysis and pay for it. For a lot of patients, this eliminates the worry of having the disease and the need for repetitive screening.

I think the biggest problem is in screening the patients when you don't really know yet what disease they have or what mutation will be found eventually-trying to round up all those first-degree relatives and then continuing to screen them over time. We have really gone more toward magnetic resonance screening for our patients in the long-term follow-up.

Dr Kouchoukos. Magnetic resonance imaging is more expensive than CT. Is there still a role for computed tomography?

Dr Braverman. Yes. I think there is certainly still a role for computed tomographic scanning. It is faster and less claustrophobic. We can do noncontrast imaging for acceptable patients whose aortas aren't particularly tortuous and get very accurate measurements.

Dr Kouchoukos. Are there any questions from the audience regarding these issues?

Dr Harold G. Roberts (Fort Lauderdale, Fla). I would like to ask a different question, specifically of Drs Mack and Svensson: 
What would they predict is the future of the transaortic valve endovascular aortic repair versus the transapical approach?

Dr Mack. I think none of this is really known yet. One of the concerns about the transapical approach is that it is more invasive than the transfemoral, definitely. And there is a fair amount of thought that the transaortic or direct aortic approach, even though it still involves a thoracotomy or partial sternotomy, may be less invasive than the transapical. So I think the experience is too small, really, to say for sure. If the transapical approach is going to have a significant role long term, it has to become percutaneous, and I think there are a number of closure devices that are out there now that are going to offer that opportunity.

But maybe, before I let Lars answer, I would ask Rudi, because he has experience with all the approaches here.

Dr Lange. As I said in my talk, the transaortic approach wasfor us - the last resort, but now there has been a revival of transaortic access, especially here in the United States. We don't favor the transaortic approach. I think it is more invasive and we would rather go for the transapical. If you are used to the transapicalonce you have done a considerable number of patients-I think it is a very safe route of access. So I don't really see any advantage of the transaortic route. In terms of strokes, it has not been shown thus far that there are fewer strokes with the transaortic than with the transapical approach. Also, as Mike pointed out, there are transapical closure systems now, and the trials will start in Europe soon. I think, in the future, we will have some kind of endoscopic procedure to access the apex, and then we won't have to do any thoracotomy at all.

Dr Svensson. I think it also is very dependent on what your patient has. As Mike said, if you have a patient who is on $4 \mathrm{~L}$ or $6 \mathrm{~L}$ of oxygen and you are doing an anterior thoracotomy, there is a price to pay for that in those high-risk patients. In the patients with really bad lung disease, I have tended to go transaortic because I think it is a quick procedure; it is very accurate, it is very easy to do, but it is obviously off label. In the patients who are having reoperations and those with porcelain aortas, then I favor-obviously-a transapical approach. We have had very good results with the transapical approach and we think it is an excellent way of doing it, but the transaortic patients recover very quickly and have minimal interference with breathing.

The other option is the anterior thoracotomy on the right side, mini anterior thoracotomy, and we have used that also in patients undergoing reoperations. They obviously are going to have a computed axial tomography scan to make sure the aorta is on the right side of the midline, and then that is a reasonable approach.

With all these approaches, including also subclavian, innominate, and carotid artery approaches, you have a lot of options; you choose what is best for your patients. There is, I think, a difference in stroke, and so you need to discuss that with your patients. And whether the transfemoral approach will continue to be dominant I think remains to be seen with future studies.

Dr Kouchoukos. Lars, you have already commented about the cost of the valve and the relative costs of these procedures versus open aortic valve replacement. Could you tell us a little bit about the relative cost of these 2 procedures at your institution?

Dr Svensson. We did a cost analysis in 2010, specifically of the cost of the transapical versus the transfemoral procedures. This was the actual cost, not charges to Medicare or Center for
Medicare and Medicaid Services (CMS) or whatever insurance company was paying for it. We were losing $\$ 4000$ for every transapical case and $\$ 3000$ for every transfemoral procedure. So those are the harsh economics. Now the price of the valve has gone up, which has increased the cost, and we have decided as an institution that we are prepared to bear the expense. We feel that we will continue to do what our conscience says is right for the patient. We, fortunately, have the ability to carry the loss, but I know that the University of Pennsylvania has done an analysis that shows its loss on each valve is even higher. So it remains to be seen how this eventually works out, especially for a commercial site.

Mike Mack, and I have spent a fair amount of time trying to work with CMS. Initially, they were only going to reimburse us at the price of a balloon valvuloplasty for the percutaneous valves - and that included the price of the valves - so that was hopeless. At least now the diagnosis-related groups (DRG) is the same as for an open AVR.

On the professional side — negotiating with the help of Mike and Peter Smith on the Society of Thoracic Surgeons coding committee- the way it is going to work is that, for transfemoral cases, the payment will be $125 \%$ of an open AVR, split between the cardiologist and the surgeon. As perhaps you have seen from the draft CMS manuscript, there has to be a surgeon and a cardiologist involved in each case.

As a final comment, if you are going to work well with your cardiologists, I think you need to put the money in one piggy bank, lockbox, or whatever you want to call it, so there isn't a battle about the professional fees over who gets the money. That will make a much more well-functioning team, which I think is critical to make this a success.

Dr Kouchoukos. What about the cost compared with just a conventional aortic valve replacement? If there is a substantial difference, is this going to have any impact as we move to patients at lower risk who are being considered for either procedure?

Dr Svensson. I agree. That is going to be a challenge. As you know, there is a geographic adjustment for DRG across the country. So, for example, in New York City, the DRG for an open AVR is about $\$ 93,000$; at the Cleveland Clinic, it is about $\$ 34,000$. So we have those parameters to work with, and the percutaneous valve currently costs $\$ 32,500$. How the economics play out is going to be very interesting for the next few years, and it is going to be very dependent on CMS. As we go into the lower risk population, we are going to see many more young patients.

Dr Paul Achoun (Paris, France). I have 2 questions. The first is for Dr Kari. As we have seen Dr Schäfers present, when you repair a bicuspid aortic valve, you have a better long-term result when you turn it into a symmetric $180^{\circ}$ configuration. So, Dr Kari, when you do your reimplantation technique in a bicuspid aortic valve, do you try to impose a $180^{\circ}$ configuration on your valve or do you just respect the natural ratio of the bicuspid valve?

Dr Kari. Thank you for this interesting question. As far as I know, Dr Schäfers and Dr Aicher looked at valves that started out with large angles. As far as I know, you looked at large-angle bicuspid aortic valve (BAV) preoperatively and did not analyze the effects of changing that angle from pre- to postoperatively. So I think there are no data on that. We tested that in our model. We didn't find any positive effect of changing that angle from small 
to large. Although we looked at those angles, the purpose of the procedure itself was not to increase the angle.

Dr Schäfers. Can I perhaps correct a misimpression? The angle that we analyzed was the angle with which the patients left the operating room, not the preoperative angle.

Dr Kari. But do you purposely enlarge the angle?

Dr Schäfers. Yes. Ever since we recognized the impact of commissural orientation, we have made an effort-whenever we replace the aorta (whether with sinotubular junction remodeling or root replacement) — to increase the angle toward as much symmetry as possible. Even if you achieve $160^{\circ}$, that is already an advantage in terms of durability compared with the angle of $140^{\circ}$, or sometimes even $130^{\circ}$, that we see on occasion.

Dr Svensson. I think the critical aspect here is the height of apposition of the leaflets. That's what you really have got to work on, apart from not narrowing it down so you end up with aortic valve stenosis. We just analyzed 780 of our bicuspid valve repairs and what is interesting is that, after the initial 1-year period, what determines late outcome is a progressive increase in gradient over time; it is virtually a linear relationship. So when you do your repair, you need to be very careful that you end up with a valve that is not stenotic, because it if is stenotic, the gradient is going to increase over time.

Dr Achouh. Thank you. My second question is for Dr Schäfers. When you do your remodeling technique plus annuloplasty, you stabilize the sinotubular junction as well as the annular junction, the annular size. In your long-term follow-up, have you ever seen any aneurysmal dilatation of the subcommissural part of the aorta, especially in Marfan patients?

Dr Schäfers. No, not yet. However, I have to admit that our follow-up in using this type of procedure for connective tissue disease is shorter than that of Dr David. We are still carefully following the patients. Up to a mean follow-up of 6 years, they are identical. We submitted a paper to the Journal of Heart Valve Disease, and it should come out soon, showing the results of both approaches. Of course, for these patients it is important to have 10- and 20-year follow-up, so I think we need to discuss this question again in 10 years.

Dr Jason Sperling (Ridgewood, $N J$ ). I have 2 quick comments and a question. There is a question about CT versus MRI for surveillance or even initial imaging. I just wanted to point out that I think that there are going to be continued innovations in imaging and its impact on how we approach the care of patients with aneurysms. Specifically, in our own program, we are using a technique called retrospective gating that has increased our diagnostic yield of type I bicuspid aortic valves dramatically. I think that is pretty exciting.

I wanted to comment to Dr Kari regarding the late finding of progressive aortic insufficiency after root replacement. I saw this initially, also, after using 36-mm grafts for the roots, and I think it is because these Dacron grafts dilate between $10 \%$ and $15 \%$ during the early postoperative period. That may be why you are seeing early dilatation. When we reduced the size of our root graft - to $34 \mathrm{~mm}$ and sometimes to $32 \mathrm{~mm}$ - we did not see that so much anymore.

My question is for Dr Milewicz. I was wondering whether you thought that there is any danger in assigning risk simply based on finding genetic mutations in these patients. I'm the furthest thing from a geneticist, but I feel that at some point we are going to be able to identify some genetic abnormality or difference in every patient with aneurysm, and I wonder whether it is a little premature simply to assign risk based on finding a gene mutation.

Dr Milewicz. There are clearly genetic variants that confer a high degree of risk, and those include the mutations in FBN1, the TGFBR1, and 2 other mutations. So I think there is a group of genetic variants that really do clearly define a high risk for thoracic aortic disease.

At the same time, within the FBN1 gene, there are genetic variants that confer no risk for aortic disease. And so it does get very complicated when you are trying to analyze genetic data. And then, if you take a gene like $M Y H 11$, there are clearly variants that cause thoracic aortic disease and other variants that do not. So I do think that we have to be careful as we analyze these variants and make sure that we are dealing with a disease-causing variant. Geneticists know how to do that analysis, and that is why I urge you to send your patients to a geneticist for genetic testing; it is not as easy as ordering the test and understanding the results immediately.

Dr Svensson. Dianna, if I could just pose one question, which we have talked about a little in the past: There are a lot of professional athletes who are very tall-I have had 4 National Basketball Association and a couple of National Collegiate Athletic Association players-who don't look as though they have Marfan syndrome, but have big roots and require operation. Any thoughts about what we should do about these patients with regard to genetic testing? It obviously has huge implications for these tall men in sports.

Dr Milewicz. That is a tough question. I do think that these athletes do need to be tested for the known genes. I think that there are a still a lot of marfanoid plus aneurysm genes that we haven't identified yet. The testing is recommended in part because of the risk for other family members and in part because it does help determine management.

Dr Kouchoukos. Let's get back to the question about sizing of grafts. Tirone, maybe you can comment on this. You have had several different ways that you determine the size of grafts when you do a valve-sparing procedure. Tell us what you do currently.

Dr David. I try to estimate the diameter of the sinotubular junction and go from there.

Dr Kouchoukos. So if the sinotubular junction measures 22 $\mathrm{mm}$, what would you pick for a graft?

Dr David. A 22-mm graft, but not for reimplantation in someone who has a 22-mm sinotubular junction.

Dr Kouchoukos. So, essentially, now you don't use any of your formulas. You just estimate the size of the sinotubular junction?

Dr David. I do not. But I do worry about the size of the annulus versus the height of the cusps. There is something I call, in the operating room, mismatch between the sinotubular junction, the annulus, and the size of the cusps. You don't have to be a mathematician to realize that if the annulus is larger than twice the height of the cusps, something is badly wrong with the valve. There is a mismatch between the size of the cusps and the size of the annulus. So you have to reduce everything to compensate for the size of the cusps. So I do not ignore the size of the cusps.

Patients with very small cusps are usually elderly, with sinotubular junction dilation as a primary mechanism of aortic 
insufficiency. Those patients require a simple operation, either only adjustment of the sinotubular junction or, at the most, a remodeling, and they end up with small grafts-22-mm or 24-mm grafts.

Dr Kouchoukos. Now, for the reimplantation procedure, you commented on the fact that you used cylindrical grafts more commonly now. You used to use larger grafts and pleat them and tuck them and so forth. You don't do that anymore?

Dr David. No, I don't. It results in more bleeding in the operating room, from more needle holes in the graft. And to be quite honest, the longest follow-up we have is in cylinder grafts, and we had the best outcomes. And, as I said only in passing, it looks as though the younger the patient when we do the operation, the better the long-term outcome, likely because the cusps may have an adaptive mechanism in response to a rigid root that is greater than in a patient who is 60 years old and has cusps that are no longer elastic.

Dr Kouchoukos. I would like to show just one slide here for a moment, if I may, that relates to what you are talking about.

This is a patient who initially was thought to have Marfan syndrome. We operated on her 15 years ago and did the David procedure. She was subsequently found to have Loeys-Dietz syndrome, and about a year ago, 15 years after this procedure was done, she sustained a dissection after pregnancy and had to have a repeat procedure. This is a photograph of that implanted valve at 15 years; the leaflets are absolutely perfect, and this is in a cylinder graft. Can I ask the other panelists here about their techniques for sizing grafts when they are doing the reimplantation procedure?

Dr El Khoury. What we do to size is quite simple. I know from echocardiographic studies done by Schäfers' team that the sinotubular junction diameter equals the height of the sinus of Valsalva, more or less. So, after deep dissection of the aortic root, I draw a line between the nadir of the left sinus and the sinotubular junction, and only size the height of the posterior commissure-30 $\mathrm{mm}, 32 \mathrm{~mm}, 28 \mathrm{~mm}$ - and I take a graft $30 \mathrm{~mm}, 32 \mathrm{~mm}$, or 28 $\mathrm{mm}$ in diameter.

Dr David. But the size corresponds magically to the sinotubular junction. It is the geometry of the cylinder.

Dr El Khoury. Exactly. For me, the sinotubular junction is the most important thing. One way to size the sinotubular junction is to size this height, which is very objective.

Dr Kari. The formula introduced a couple of years ago by $\mathrm{Dr}$ Feindel and Dr David - taking the aortic lumen multiplied by 2, then multiplied by 0.67 , and then adding twice the tissue thickness - is used as a complement to the intuitive surgeon's choice, I think. It is used at Stanford.

Dr Kouchoukos. Dr David mentioned that he doesn't use the Valsalva graft. Do you want to tell us your feelings about the Valsalva graft? And do any of the other panelists use a Valsalva graft in this procedure?

Dr David. Take a look at the anatomy. The aortic root is not spherical. The aortic root is a cylinder with 3 spheres or hemispheres to make the sinuses. Gebrine El Khoury had a beautiful slide of a cadaveric root with no sinuses. What shape was it for Gebrine? A cylinder. Read all the work that Francis Robicsek and Mano Thubrikar published 20 years ago on the anatomy or the geometry of the aortic root -3 cusps within a cylinder with 3 bulges. The Valsalva graft is a sphere. You are deforming the aortic annulus. I'll bet you 10 years from now that we are going to be sitting here and saying he was right.
Dr Kouchoukos. Does anybody on the panel use the Valsalva graft?

Dr El Khoury. I use the Valsalva. The way you work on the Valsalva tube-by scalloping and aligning the commissures to the Valsalva-can help us to make these 3-piece sinuses. If you put it in the way it is, as a cylinder, you will have a cylindrical root, but if you work on aligning it-

Dr David. But he is plicating in so many places. It's worse than a dog's breakfast to make a cylinder. So use a cylinder and plicate the sinuses.

Dr El Khoury. When you are doing the second plane, it is very easy to work with the Valsalva. When you want to place the 3 commissures, it is very easy, because there is a lot of discussion of how far we should extend the straight tube or whatever. The Valsalva is easier to work with.

Dr Kouchoukos. Let's ask the others. Dr Schäfers, Dr Svensson, Dr Mack, what is your preference?

Dr Mack. I use it, and I am afraid to admit it right now, but I feel better because Rudi uses it also. Do you want to comment, Rudi?

Dr Lange. I don't dare to get into another fight with Tirone, of course, because we have discussed this many times. We use the Valsalva graft exclusively and, altogether, we have about 200 patients now-maybe 50 have a straight tube and 150 have a Valsalva graft. And to date, we don't see any difference at all in the longterm outcome.

Dr Svensson. I have had to reoperate on a couple of patients with the Valsalva grafts, so I tend to lean toward what Tirone has been saying. I have seen these patients come back with 4.5 to 4.7-mm roots; the leaflets get pulled apart. I don't use it and I haven't used it for that purpose. I think it is a great graft for biologic composite valves.

As far as sizing, I looked at Tirone's paper and wondered: Am I really going to calculate this on every patient? And then I saw he was using $30-\mathrm{mm}$ tube grafts for most of his patients. And so that is what I use for most patients. I use a $28-\mathrm{mm}$ graft for a smallish woman, and for a professional player or someone like that, I may go up to $34 \mathrm{~mm}$. And the other thing I do is use the formula based on body surface area and insert a Hegar. I think that is much more important as far as long-term durability. You want to have at least 3 to $5 \mathrm{~mm}$ of apposition to maintain good function of those leaflets.

Dr Schäfers. I don't use the Valsalva graft. My feeling-and this is intuitive - is that the operation must include optimal height of the commissures. You have to place them as high as possible. I have done a number of reoperations from other centers where commissural height was the primary problem. The Valsalva graft limits, by definition, the highest point at which I can place the commissures. This is why I prefer the tube graft, because it is unlimited. I can simply place the commissures as high as I want.

Dr Kouchoukos. Good point.

Dr Francois Bouchart (Rouen, France). I want to ask a question about aortic root dilation. If you have a young woman, 16 to 18 years old, with Loeys-Dietz syndrome and an aortic root of $40 \mathrm{~mm}$ or $42 \mathrm{~mm}$, you plan to change it. Where do you stop on the aorta? We have good results with surgery of the aortic arch, especially in a nonacute setting, and why not replace the aortic arch? Would it seem unreasonable?

Dr Kouchoukos. Duke Cameron, do you want to respond? 
Dr Cameron. There isn't really enough experience to comment on that. I did mention in my talk that in the younger Loeys-Dietz patients in whom we didn't replace even the entire ascending aorta, there was a small remnant of distal aorta proximal to the innominate with some dilatation that we really haven't seen frequently in Marfan patients. So I think a more aggressive replacement of the aorta makes some sense, but I think you have got to be able to do an arch replacement with a very low operative risk to justify an approach like that.

Dr Ziad Hanhan (Jersey City, NJ). I have a clinical question, a hypothetical situation. A 30-year-old woman with a type A dissection that is limited to the ascending aorta has a bicuspid aortic valve, and the aortic root is $4.5 \mathrm{~cm}$. Which operation would you perform? Would you go for the Bentall prophylactically or just replace the ascending aorta, if you could?

Dr Schäfers. In a small woman, a $4.5-\mathrm{cm}$ root means that it is markedly enlarged. There are no published prognostic data showing the degree or the likelihood of root dilatation in that patient. On the other hand, if we index it for patient size and then apply common sense, I think one can make a strong argument in favor of root replacement, whether it is a Bentall replacement or maybe a valvepreserving replacement.

Dr Hanhan. Just to follow up, is that a consensus for the community cardiothoracic surgeon who can do a Bentall but also could lower the risk by just replacing the ascending aorta in an acutely dissected situation? I'm just trying to get some controversy here.

Dr David. Save the patient's life. Do the simplest possible operation so she goes home alive, and then send her to somebody else to do a valve-sparing operation later on.

Dr Luca Botta (Milan, Italy). I have a question for Drs El Khoury and Schäfers. I would like to know from them if there is still a role for selective replacement of the noncoronary sinus in a bicuspid valve in a patient with dilatation of the ascending aorta and a bicuspid valve.

Dr Schäfers. While Gebrine is thinking about the answer, I think-but this is completely personal-no. Why don't I do it? We don't have good information on the ascending aorta, but we know from the aortic isthmus that whenever we introduce prosthetic material in an asymmetric fashion-and I am thinking of the patch plasty that was done 30,40 years ago for coarctation repair-this resulted in a relatively high incidence of aneurysm formation. For that reason, even though at times it looks like the noncoronary sinus is the main problem, I prefer to do a symmetric operation-that is, replace all sinuses.

Having said that, it is often proposed that 1 sinus is larger than the others and the operation should maybe include only that 1 sinus. At this time, I am not aware of any long-term data showing that there is a disadvantage in doing it.

Dr El Khoury. I agree totally, but I think one of the first things to think about is the age of the patient. If the patient is quite old50,60 years-I think replacing the noncoronary sinus can be a good idea, because the aortoventricular junction is stable and there is no risk for further dilation at the level of the aortoventricular junction. If the patient is young-20, 25, or 30 years-I think we should be more aggressive and do a symmetric reconstruction of the aortic root.

Dr Kouchoukos. I would like to ask Dr Lange: You showed some data indicating that the percutaneous valves now are approximately $30 \%$ or so of all the valve replacements in Germany. Has that reduced the number of open aortic valve replacements or are these in addition to the standard aortic valve replacements?

Dr Lange. The statistics show very clearly that percutaneous valve surgery has reduced the number of open procedures. This trend started about 2 years ago. There was a plateau for 2 years, and now the number of open procedures is decreasing.

Dr Kouchoukos. By what percent would you estimate?

Dr Lange. I would say by perhaps $10 \%$.

Dr Achouh. I would like to ask Dr Milewicz: In the setting of an aortic surgery program, do you recommend genetic counseling whenever a patient comes in with an acute dissection or do we have to have some family history of dissection or something else?

Dr Milewicz. I think if the patient is young or there is a family history, genetic counseling is warranted. The treatment guidelines state that you should work up the family members, but that can be done by the cardiologist.

Dr Achouh. If we don't have any apparent family history, according to the patient, do you still send him for genetic counseling?

Dr Milewicz. I would if the patient was young, $<50$ or 55 years.

Dr Hitoshi Ogino (Suita, Japan). My question is to Drs David, El Khoury, and Schäfers. My concern is about cusp repair technique. There are actually 2 techniques: central plication and another technique of reinforcement, which Dr David mentioned, as well as resuspension, to which Dr El Khoury alluded. For what cusp pathology are both techniques suitable?

Dr David. The video I showed you was representative of a normal cusp. If the cusp is normal in thickness, I think plication is the way to go. However, if the free margin you can see is overstretched, is paper thin, or even thinner than paper, then I reinforce with a double layer of Gore-Tex, one very close to the free margin, and a second layer of Gore-Tex a few millimeters below.

Two years ago when I gave a talk on this here, I showed a video of how we do it, and I showed the result of a patient who had had Gore-Tex 13 years earlier and came back for reoperation because of mitral valve repair, in whom we explored the aortic valve. You could not see the Gore-Tex anymore. It is identical to Gore-Tex in the mitral chordae tendinae. It becomes totally covered by fibrous tissue and reinforces the free margin. I think Gebrine, whom I trained 15 years ago, took this to a new level. To be quite honest, I was very conservative initially. By watching him do it more freely, I expanded the indications as well. And they have published this; in $>100$ cases now, reinforcement with Gore-Tex seems to stabilize the cusps. By doing this, you expand the indication of aortic valve sparing, particularly in younger patients-people $<30$ or 40 years of age, in whom you don't want to put a mechanical valve and keep them on anticoagulation for a lifetime.

Dr Kouchoukos. Duke, let me ask you a question that relates to this. What is your current indication to use a mechanical valve in a patient with either Marfan or Loeys-Dietz syndrome? When do you decide to use a mechanical prosthesis and a composite graft?

Dr Cameron. Essentially, only if the patient has requested it up front. We still have some patients who have other family members who have composite grafts who have done well with them, and they actually ask for a mechanical valve. The other patients would be those who already have a mechanical mitral valve. But most of 
the patients who are not candidates for valve sparing usually ask for a biologic Bentall as their backup.

Dr Kouchoukos. Have you had to do repairs on any of the leaflets in this group of patients?

Dr Cameron. In the Marfan group, it is about $10 \%$ to $15 \%$, and in our group of patients, the commissures are so thin that we have really not been able to do free-edge suturing, so we will do a midleaflet plication. I think only 1 of those Loeys-Dietz patients had a midleaflet plication.

Dr Manu N. Mathur (Sydney, Australia). With the valve-sparing roots, most of the patients have mild or minimal aortic insufficiency (AI), because in the patients with root aneurysms with severe AI, you look at the leaflets and you think that this is not going to give a good long-term result. I would like to know from all the surgeons on the panel what proportion of their cases when they are doing a valve-sparing root actually have severe AI.

Dr David. You saw in our slide that approximately half of our patients had moderate AI and one fourth had severe AI at the time of the operation. I don't think severe AI necessarily means bad cusps in patients who have aortic root aneurysm. It means the root is characterized by what I said before: there is a mismatch between the surface area of the cusps and the orifice of the cusps. Some cusps tolerate it very well; other ones fall apart.

I don't want to convey the message that I repair every aortic valve, by a long shot. We still do 4:1 Bentalls in Toronto versus valve sparing. So it is not that common. These are highly selected patients. But if the cusps are prolapsing, we fix them if the substance is reasonable.

Dr Mathur. If the roots are $>6 \mathrm{~cm}$, are you still doing valve sparing?

Dr David. Even $70 \mathrm{~mm}$ or $80 \mathrm{~mm}$. It doesn't matter. It all depends on the cusps. Remember, sinus of Valsalva dilatation does not cause aortic insufficiency. What causes aortic insufficiency is annular and sinotubular junction dilatation. You can rupture the sinus of Valsalva into the right ventricle. Like children, they have a competent aortic valve and there is a hole in the sinus, and the valve stays competent. So you can have a sinus of Valsalva as big as you like. The 2 things that play on the function of aortic insufficiency are the sinotubular junction and the diameter of the aortic annulus.

Dr Mathur. You said in Toronto it's 4:1, root replacement versus valve sparing.

Dr David. We do about 120 Bentalls a year.

Dr Mathur. Why aren't they having valve-sparing roots? Why aren't they suitable?

Dr David. They are bicuspid, calcified; they are bad aortic valves. We can't repair some of the Marfan valves; the cusps are all destroyed. In those, of course, you replace them. The whole key is the quality of the cusps. If the cusps are of reasonable quality, I think it is a good and lasting operation.

Dr Schäfers. In our experience, roughly $70 \%$ of the patients have either grade 3 or grade 4 aortic regurgitation, maybe even a little more. I don't have the exact figures. I think it is a good question, and so far as a rule of thumb, if there is minimal regurgitation, there is also minimal alteration of cusp geometry. If there is relevant aortic regurgitation, there is a very high likelihood that you end up with prolapse of at least 1 if not more cusps. So when there is severe aortic regurgitation, and you go for a valve-preserving operation, you must expect cusp prolapse. You must familiarize yourself with the techniques of assessing cusp geometry and of correcting the prolapse.

Dr Svensson. I would add to what Tirone is saying that as far as size and regurgitation amount by themselves, those are not reasons not to do a David reimplantation. In our population, we looked at this a while ago: $5 \%$ of our patients had $4+$ AI. So that is not a reason to exclude a patient.

The big question is whether there are fenestrations in the leaflets, and how big they are. So, as Tirone was saying, leaflet quality is paramount. If you have good leaflet quality, then you can always repair the valve if need be, and then do a David reimplantation; you don't have to replace the valve. So, as other people have said, it takes judgment over time to decide in which patients you can keep the leaflets.

The other thing I would say as a rule of thumb is that in those patients with more than 2 leaflets that are prolapsing, you probably shouldn't try to do a David. It usually doesn't work out. It is very easy to correct the prolapse if there is one leaflet prolapsing. With 2 leaflets, you can hitch up 1 commissure and reimplant at the higher level, but when you have 3 leaflets prolapsing, then it usually is not going to work.

Dr Said A. B. Soliman (Cairo, Egypt). My question is for Drs El Khoury and Schäfers about patients with bicuspid aortic valve. Sometimes we are faced with patients who have mild stenosis, not insufficiency alone, with a gradient of $40 \mathrm{~mm}$ or maybe $50 \mathrm{~mm}$. In such cases, do you proceed with valve sparing when there is a mild stenosis and some calcification of the leaflets? A second question also is about using annular stabilization. Is there is a difference between the Gore-Tex stitch and the annular ring?

Dr El Khoury. I think for the gradient, the whole issue is the quality of tissue. You can have a normal bicuspid aortic valve with a huge dilation of the aortic root and a big gradient. Now, if you do sparing surgery, the gradient will disappear because you have more motion of the leaflet.

The question is different if the leaflets are calcified. I was quite aggressive some years ago; now I have become a little bit less so. If the bicuspid valve is calcified, I will replace the valve unless it is really localized calcification on the raphe or at the base of the leaflet. So a gradient, per se, is not really a contraindication to valve sparing, providing that you have good quality of tissue. If there is no calcification, we go ahead.

Regarding annuloplasty, for both bicuspid and tricuspid valves, my preferred technique for stabilization is reimplantation. I have never tried the Gore-Tex stabilization. The only comment I can make is that when we are repairing the bicuspid valve, we like to have what I will call selective annuloplasty of the anterior part of the bicuspid annulus. I am not sure, and I will ask Joachim for his comment, but with a circular annuloplasty, there is no selective effect. We are reducing the whole circumference. I don't know whether I am right.

Dr Schäfers. Let me answer the second question first. At this point I have not seen a disadvantage in terms of root geometry of the circular annuloplasty via a suture. Early on, we had a couple of complications. This was when we used Ethibond, and we had 2 or 3 patients with secondary erosion of the membranous ventricular septum. This is something that, of course, you will not expect or 
cannot expect when you do a David operation. This problem seems to have been largely eliminated just by changing the material.

On the other hand, I said these are early results that I presented today, and probably we are not dealing with the ideal implant yet. My vision is to have an implant that you can pass through muscle that has a bigger diameter than a suture and therefore better stress distribution.

To come back to your question regarding the gradient, I had a similar experience in the past. I thought it was very easy to cut out the calcium, suture in a patch, or just peel off calcium if there was a calcific bar. I have become less enthusiastic about this. Insertion of a patch into, for instance, the area of the median raphe is an independent predictor of failure, and I have become very conservative regarding insertion of patch material in a bicuspid valve. It is different for a tricuspid valve. The only 2 patients on whom I had to operate for aortic stenosis 10 years and 11 years after remodeling a bicuspid valve were patients in whom I peeled a limited calcific bar off the noncoronary cusp at the initial operation. Still, the operation had a durability of 10 or 11 years. At reoperation, the valve was completely calcified. So, probably, a bicuspid valve that already exhibits calcifications at the time you touch it is not a good substrate for valve preservation.

Dr Soliman. So you proceed, from the start, to change these valves?

Dr Schäfers. Yes. Today, if I were to operate and see calcifications, if it were a very young patient and I could get by with just resecting the calcium and making the aorta smaller, and I still would have enough tissue left, I would go for a direct readaptation. If there were more extensive calcification, I would strongly recommend replacement.

Dr Jean E. Bachet (Abu Dhabi, United Arab Emirates). The previous question prompts me to ask Gebrine and Hans Schäfers the following questions. When we see your results and your video, it seems very simple to repair those valves, but I am convinced that it is not so simple; otherwise, it would be much more popular. And you are speaking about decalcifying, etc. What exactly is the proportion of the patients with an aortic valve problem that you repair and that you replace in your department?

And you said, for instance, that the quality of the valve, in your own words, is the only criterion. How do you appreciate that? Is it just that you look at the valve and say, "This is a good one; this is a bad one?" In other words, what are your objective criteria to decide whether you will replace a valve or repair it?

Dr Schäfers. The proportion of repair in our total volume is $37 \%$ to $38 \%$. It was up to $40 \%$ a few years ago, and then we became a little more conservative in some of the bicuspid valves. Objective criteria? One is calcification, which we have already commented on. Dr Svensson also mentioned the presence of multiple fenestrations. My feeling is that 1 or 2 fenestrations can be closed, although it is a more complex procedure. But if there are 3,4 , or 5 fenestrations, I think that valve is better replaced.

In addition, there is the important and interesting question of cusp retraction in rheumatic valve disease, which I think creates a suboptimal substrate for repair. Currently I am fighting through the editorial comments concerning a publication that I submitted to the Journal of Thoracic and Cardiovascular Surgery that looks at cusp height — at what I call geometric height, just the amount of tissue. A normal tricuspid valve has a cusp height of $20 \mathrm{~mm}$. If it is
$17 \mathrm{~mm}$ in an adult patient, this cusp is already retracted, and for the bicuspid valve it is similar. For the 1 large cusp, the mean is 23 $\mathrm{mm}$, and if it is $20 \mathrm{~mm}$, it is borderline; if $<20 \mathrm{~mm}$, that amount of tissue is so little that I would recommend not repairing the valve, considering the cusp to be retracted. With these valves we would then go for replacement.

Dr El Khoury. I agree totally. The only thing that I can say is that if you have an aneurysmal problem, and you have the echocardiographic and surgical assessment that the valve is normal, it can be preserved in $100 \%$ of patients.

In a patient with aortic regurgitation, the problem is a little bit different. It depends on the quality of tissue, for sure, but what is really more important is the quality of tissue at the end of surgery. Let's say there is a small calcification, a small thickening, or something like that, and you can resect it and restore good motion of the leaflet, and at the end of the surgery we have good results-

Dr Schäfers. Give us a number. By the way, for us, the rate of repair is $40 \%$ of total volume; $85 \%$ for aortic regurgitation. Gebrine, you say the same numbers. Total volume repair and repair in regurgitation-give us the percentage.

Dr El Khoury. Eighty to $90 \%$.

Dr Bachet. Does age enter into your decision? In other words, if you have a patient who is 70 years old, would you spend time repairing his valve instead of putting in a nice pericardial valve, which will take 45 minutes and which will last 20 years?

Dr El Khoury. It depends. If it is a dilated root, you will do a Bentall. There is a big difference in time between Bentall and valve-sparing surgery.

Dr Bachet. I am not speaking of dilatation of the root. Don't change the subject.

Dr El Khoury. If you have only prolapse of the noncoronary, of the right, I think you can finish the operation also with 2 stitches. If you are confident in your technique, you can do a repair; you can do whatever you decide. The major thing is to be confident in your surgery.

Dr Carlo Antona (Milan, Italy). Reinforcement of the free margin with Gore-Tex is an excellent technique, but I think that, today, we can use it only for anatomic stabilization, but not for the physiology of the valve. We have demonstrated that when you obtain good coaptation, the free margin is completely destressed, and this is the reason you can plicate-only plicate-for a short prolapse without reinforcing the free margin. I have had excellent results. We can reinforce the free margin to resolve a small degree of prolapse, but not to stabilize the valve. It is only an anatomic stabilization, because with finite elements we show that the valve is completely destressed. It means that, in my view, the most important problem is to have nice coaptation, more than $4 \mathrm{~mm}, 5$ $\mathrm{mm}, 6 \mathrm{~mm}$ of coaptation.

Dr David. I agree entirely. You restore anatomy first, and then if there are 2 or 3 large fenestrations you are concerned are going to rupture, it doesn't matter how good a coaptation you have. My slide shows well a very large fenestration that extends to the central portion. That kind of cusp should be repaired or the valve replaced. So, yes, one should use plication of the central portion to repair prolapse, but in addition to that, if the cusps are of bad quality, reinforce with Gore-Tex-7-0 Gore-Tex, 2 or 3 layers sometimes. You are going to guarantee longer durability for the repair. 
Dr Antona. Some time ago, at the last meeting, we spoke about free suspension. Now, I think, something has changed—only anatomic stabilization.

Dr David. No, I never use Gore-Tex to correct prolapse. From day 1 , from my very first publication, I said I used Gore-Tex to repair large fenestrations. Prolapse you repair by plication. You can correct $1 \mathrm{~mm}$ or $2 \mathrm{~mm}$ of prolapse by suspending a bit, but if the prolapse is $0.5 \mathrm{~cm}$, you should not rely on the Gore-Tex to fix the prolapse.

Dr Kouchoukos. Our time is up. I want to thank all of the panelists for their presentations and comments, and the audience for their attention. 